Conclusion The incidence of NTM has continued to rise since the last national survey. This represents an almost ten-fold increase since 1995. The majority of these are pulmonary isolates (in particular MAI). Possible explanations include greater awareness amongst clinicians leading to increased sampling, improvements in laboratory techniques for speciation or laboratory reporting practices. However, such a large increase most likely reflects a genuine rise in NTM infection in the population. Given this change in culture confirmation, it is imperative that a comprehensive clinical database is set up to provide national monitoring of clinically significant infections, and establish the true burden of disease present in EW and NI.

\section{P189 SHOULD SCREENING FOR CHRONIC VIRAL HEPATITIS IN PATIENTS WITH TUBERCULOSIS BE INTRODUCED TO NICE GUIDELINES?}

JL Potter, C Hyams, M Shaukat, ZO Babiker, VM Macavei, N Jayasekera, H Kunst, GR Foster, VLC White. Barts Health NHS Trust, London, UK

\subsection{6/thoraxjnl-2014-206260.318}

Background Screening for viral hepatitis is not routinely recommended in patients diagnosed with tuberculosis (TB). However there are significant similarities in the global distribution of $\mathrm{TB}$ and hepatitis B (HBV) and C (HCV). It remains unclear whether co-infection with HBV or HCV is a risk factor for hepatotoxicity in patients receiving anti-tuberculous therapy and significant morbidity and mortality is associated with a late diagnosis.

Objectives To determine the prevalence of HBV and HCV infection among new cases of active TB across treatment centres in East London and to assess the adverse drug reactions to antituberculous treatment experienced by this population.

Methods We conducted a retrospective study including all patients diagnosed with active TB during 2013 at two TB clinics in London. Data on demographic characteristics, HBV surface antigen (HBsAg), HCV antibody, human immunodeficiency virus (HIV) and adverse drug reactions were retrospectively analysed.

Results In total, 472 cases of active TB were notified during 2013. The mean age was $37.7(+/-15.3)$ years (range: 5-92). Males accounted for $62.3 \%$ of our cohort. $84.7 \%$ of patients were born outside of the UK with the majority of patients being born in either Bangladesh (16.5\%), India (27.8\%) or Pakistan (15.9\%). Overall, 304 patients were screened for HBV, 302 for $\mathrm{HCV}$, and 447 for HIV. Of those screened, HBsAg was detected in $3.3 \%$, HCV antibody in $2.0 \%$ and HIV in $3.4 \%$. All patients infected with HBV or HCV were foreign born. Hepatotoxicity was defined as an ALT greater than 5 times the upper limit of normal or requiring a change in treatment. There was no significant difference in rates of hepatotoxicity in either in HepBsAg status $(\mathrm{p}=0.371)$, HCV status $(\mathrm{p}=0.597)$ or HIV status $(p=0.413)$ but numbers of HBV and HCV infection were small.

Conclusions The prevalence of $\mathrm{HBV}$ and $\mathrm{HCV}$ was significantly higher in our cohort of TB patients than the background UK prevalence, which is $0.4 \%$ for $\mathrm{HCV}, 0.3 \%$ for $\mathrm{HBV}$ and $0.15 \%$ for HIV. Routine screening for HBV and HCV on an opt-out basis would be justified in our setting given the high proportion of foreign-born patients. Further research into the magnitude of $\mathrm{HBV} / \mathrm{HCV}$ co-infection with active or latent TB, any increased risk in drug-induced hepatotoxicity and the cost-effectiveness of routine screening is needed.

\section{P190} DRUG INDUCED LIVER INJURY IN THE TREATMENT OF TUBERCULOSIS IN A BUSY UK CENTRE

${ }^{1} \mathrm{~S}$ Chitty, ${ }^{1} \mathrm{R}$ Ghani, ${ }^{1} \mathrm{JK}$ Roe, ${ }^{1} \mathrm{H}$ Davidson, ${ }^{1} \mathrm{M}$ Routledge, ${ }^{1} \mathrm{~T}$ Edwards, ${ }^{1} \mathrm{C}$ Hateley, ${ }^{2} \mathrm{~S}$ Collin, ${ }^{3}$ A Ritchie, ${ }^{1} \mathrm{~J}$ Dzvova, 'J Buckley, ${ }^{1} \mathrm{RN}$ Davidson, 'L John. 'Northwick Park Hospital, London, UK; ${ }^{2}$ School of Social and Community Science, University of Bristol, Bristol, UK; ${ }^{3}$ Department of Respiratory Medicine, Central Middlesex Hospital, London, UK

\subsection{6/thoraxjnl-2014-206260.319}

Introduction We describe the incidence and management of drug induced liver injury (DILI) in active TB at the largest UK centre, using a nurse-led local protocol derived from 1998 BTS guidelines.

Methods All active TB cases were identified from April 2010 to May 2014. Patients were identified with DILI by following criteria: Type 1 DILI(ALT $>3 \mathrm{x}$ upper limit normal (ULN-55iu/l), Type 2 DILI (ALP $>2 \mathrm{x}$ ULN(150 iu/l) and Bilirubin $>21 \mathrm{iu} / \mathrm{l})$ or Type 3 DILI (Bilirubin $>40 \mathrm{iu} / \mathrm{l}$ ). Patient demographics, TB treatment (ATT), timing, management and outcomes of DILI were described. Baseline characteristics and ATT doses were matched with controls.

Results 105 individuals with DILI were identified out of 1529 patients with active TB (6.9\%). 81\% were on standard first line therapy (Rifampicin (R), Isoniazid $(\mathrm{H})$, Pyrazinamide $(\mathrm{Z})$ and Ethambutol (E)). 7.8\% were on Moxifloxacin (M) instead of E and $1.9 \%$ were on RHME. Type 1 DILI was most frequent (81\%) with median peak ALT $296 \mathrm{iu} / \mathrm{l}$ (IQR 227-505). Median time from treatment start to onset of DILI was 12.5 days (7-30). Symptoms at presentation included nausea/vomiting (54\%), abdominal pain (18\%) and jaundice (12.4\%). 45.7\% patients had all medication stopped, $7.6 \%$ continued ethambutol with amikacin (A), $26.7 \%$ continued all medication, $6.7 \%$ stopped Z only, 3.8\% substituted Z for a quinolone. Median time from stopping to reintroduction was 10 days (6-17). Of 66 reintroduction patients, regimens included $\mathrm{H}>\mathrm{R}>\mathrm{E}(45 \%)$, $\mathrm{H}>\mathrm{R}>\mathrm{E}>\mathrm{M}(31 \%)$ and $\mathrm{R}>\mathrm{E}>\mathrm{M}(15 \%)$. Median time from reintroduction to full treatment restart was 14 days (12-18). $81 \%$ of patients were uneventfully reintroduced, $5 \%$ suffered a 2 nd DILI. 32\% patients required hospital admission and 4(3.8\%) died.

DILI cases were matched to 200 controls. Cases more likely $(\mathrm{P}<0.05)$ to; be HIV positive, have quinolones in initial regimen and lower body weight. Quinolone use gave an adjusted hazard ratio $5.41(2.96,9.91)$.

Conclusion DILI remains the most important toxicity of ATT and usually occurs during the first month. The BTS guideline provides a useful template for the diagnosis and management of DILI which may be largely nurse led and ambulatory. Most patients are successfully reintroduced without pyrazinamide. HIV status, body weight and quinolone use are risk factors.

\section{P191 WITH A LOW INCIDENCE OF DRUG-INDUCED HEPATITIS, SHOULD WE BE OFFERING LATENT TB TREATMENT TO MORE PATIENTS OVER THE AGE OF 35?}

P Howlett, N Lungu, W Owen, R Breen, L Baker. Guys and St Thomas' Hospital, London, UK

\subsection{6/thoraxjnl-2014-206260.320}

Introduction NICE guidelines recommend patients $>35$ yrs at risk of tuberculosis (TB) on screening, but without active disease, should not be offered latent TB infection (LTBI) treatment unless a healthcare worker, or HIV positive. This is based on perceived 


\section{Correction}

S Chitty, R Ghani, JK Roe, et al. P190 Drug Induced Liver Injury In The Treatment Of Tuberculosis In A Busy Uk Centre. Thorax 2014;69(Suppl 2):A159 doi:10.1136/ thoraxjnl-2014-206260.319

There are 3 corrections to this article.

1. An author was omitted and the correct author list should be the following:

S Chitty, ${ }^{1}$ R Ghani, ${ }^{1}$ A Abbara, ${ }^{1} \mathrm{JK}$ Roe, ${ }^{1} \mathrm{H}$ Davidson, ${ }^{1} \mathrm{M}$ Routledge, ${ }^{1} \mathrm{~T}$ Edwards, ${ }^{1}$ C Hateley, ${ }^{1}$ S Collin, ${ }^{2}$ A Ritchie, ${ }^{3} \mathrm{~J}$ Dzvova, ${ }^{1} \mathrm{~J}$ Buckley, ${ }^{1} \mathrm{RN}$ Davidson, ${ }^{1} \mathrm{~L}$ John ${ }^{1}$

${ }^{1}$ Northwick Park Hospital, London, UK

${ }^{2}$ School of Social and Community Science, University of Bristol, Bristol, UK

${ }^{3}$ Department of Respiratory Medicine, Central Middlesex Hospital, London, UK

2. The following sentence should be added to the end of the Results section, 'DILI cases were matched to 200 controls. Cases were more likely to be HIV positive $(p<0.05$, odds ratio 3.81) and have a lower body weight (odds ratio 0.96).'

3. The revised Conclusion section is as follows:

DILI remains the most important toxicity of ATT with peak occurrence at 12.5 days. The BTS guideline provides a useful template for the diagnosis and management of DILI which can be largely nurse led and ambulatory. Most patients are successfully reintroduced without pyrazinamide. HIV status and low body weight are risk factors.

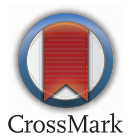

Thorax 2015;70:301. doi:10.1136/thoraxjnl-2014-206260.319corr1 